\title{
Successful use of the HeartWare HVAD rotary blood pump for biventricular support
}

\author{
Martin Strueber, MD, Anna L. Meyer, MD, Doris Malehsa, MD, and Axel Haverich, MD, Hannover, \\ Germany
}

Left ventricular assist devices (LVADs) using rotary blood pump technology led to significant improvement of results in left ventricular failure. ${ }^{1}$ However, patients with concomitant right ventricular failure have not benefited from this new technology so far. We report on the first successful use of 2 HeartWare HVAD centrifugal pumps (HeartWare International, Inc, Framingham, Mass) in biventricular support.

\section{CLINICAL SUMMARY}

A 56-year-old man was referred to our institution after massive myocardial infarction owing to occlusion of the left anterior descending coronary artery (LAD) (maximum creatine kinase MB 1300 units). He had been treated with grafts to the LAD, the first diagonal, and the right coronary artery (RCA) and was in cardiogenic shock. An intra-aortic balloon pump (IABP) and a femorofemoral extracorporeal membrane oxygenator (ECMO) were implanted after weaning from extracorporeal circulation failed. The patient was transported to our center after 2 days in an extubated stable state.

An LVAD was implanted using a standard approach under ECMO support.

All bypass grafts were intact; however, right ventricular dysfunction led to the decision not to explant the ECMO or the IABP at this time. The IABP was removed the following day, and the ECMO was explanted on postoperative day 4 when renal and liver parameters remained normal. The patient was treated with phosphodiesterase inhibitor and nitric oxide. Sedation was instituted for another 3 days, and then weaning from ventilatory support was started. At this time the compensated right ventricular insufficiency turned into refractory right ventricular failure inducing renal insufficiency and impairment of liver function. A new ECMO was inserted on postoperative day 11. Again, end-organ

From the Departments of Cardiothoracic-, Transplantation- and Vascular Surgery, Hannover Medical School, Hannover, Germany.

Disclosures: None.

Received for publication Jan 25, 2010; revisions received March 29, 2010; accepted for publication April 10, 2010; available ahead of print May 17, 2010.

Address for reprints: Martin Strueber, MD, Cardiothoracic-, Transplantation- and Vascular Surgery, Hannover Medical School, Carl-Neuberg-Str. 1, 30625 Hannover, Germany (E-mail: strueber.martin@mh-hannover.de).

J Thorac Cardiovasc Surg 2010;140:936-7

0022-5223/\$36.00

Copyright (c) 2010 by The American Association for Thoracic Surgery doi:10.1016/j.jtcvs.2010.04.007 function recovered, but right heart failure persisted as indicated by echocardiographic findings for another 10 days, and the decision was made to implant a second HVAD device to support the right ventricle. With the patient still supported by ECMO, the anterior wall of the right ventricle was chosen for implantion of the inflow of the device. The sewing ring was placed epicardially. Care was taken not to insert the whole length of the inflow cannula, but only about $1 \mathrm{~cm}$ into the cavity of the ventricle. The location of the device was stabilized by the epicardial sewing ring.

The bend relief was removed and after partial clamping the outflow graft was sewn end to side to the main pulmonary artery (Figure 1).

For continuous pressure monitoring, a catheter was inserted into the left atrium (LA). The LVAD was set to relativey high speed (3600 rpm) and the right ventricular assist device (RVAD) was started at a pump speed of $2000 \mathrm{rpm}$. The ECMO could be stopped and removed. There were stable circulatory conditions with a pump flow of 4.5 to $5.5 \mathrm{~L} / \mathrm{min}$ on the LVAD and an LA pressure between 3 and $9 \mathrm{~mm} \mathrm{Hg}$.

After the start of spontaneous breathing, the pump flow on the RVAD changed by the breathing pattern, with very high

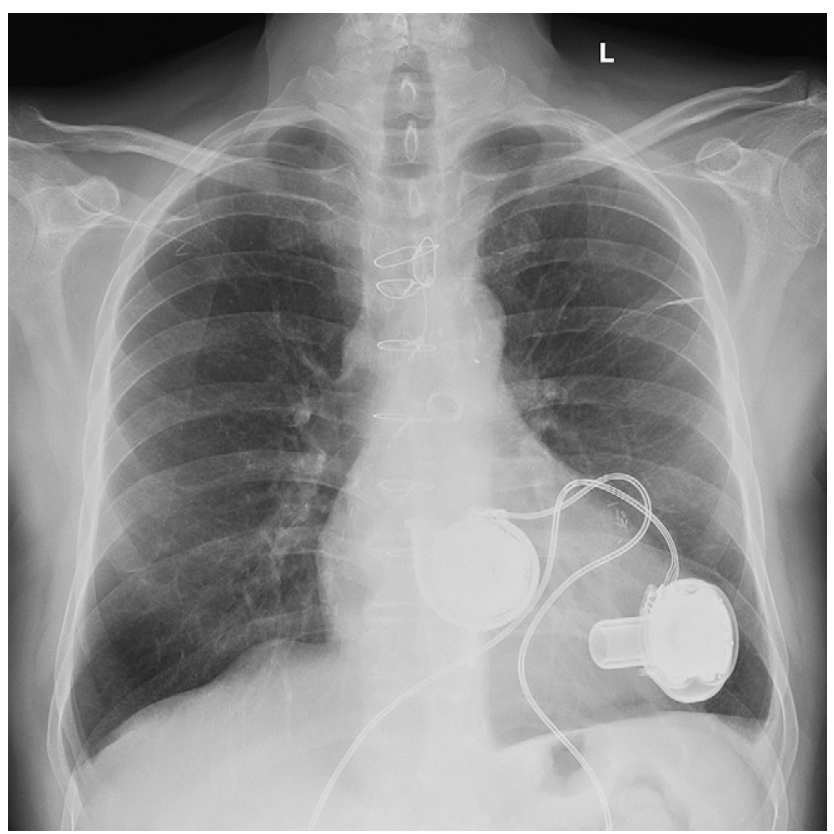

FIGURE 1. Chest $x$-ray film showing 2 HeartWare HVAD pumps in biventricular support. 


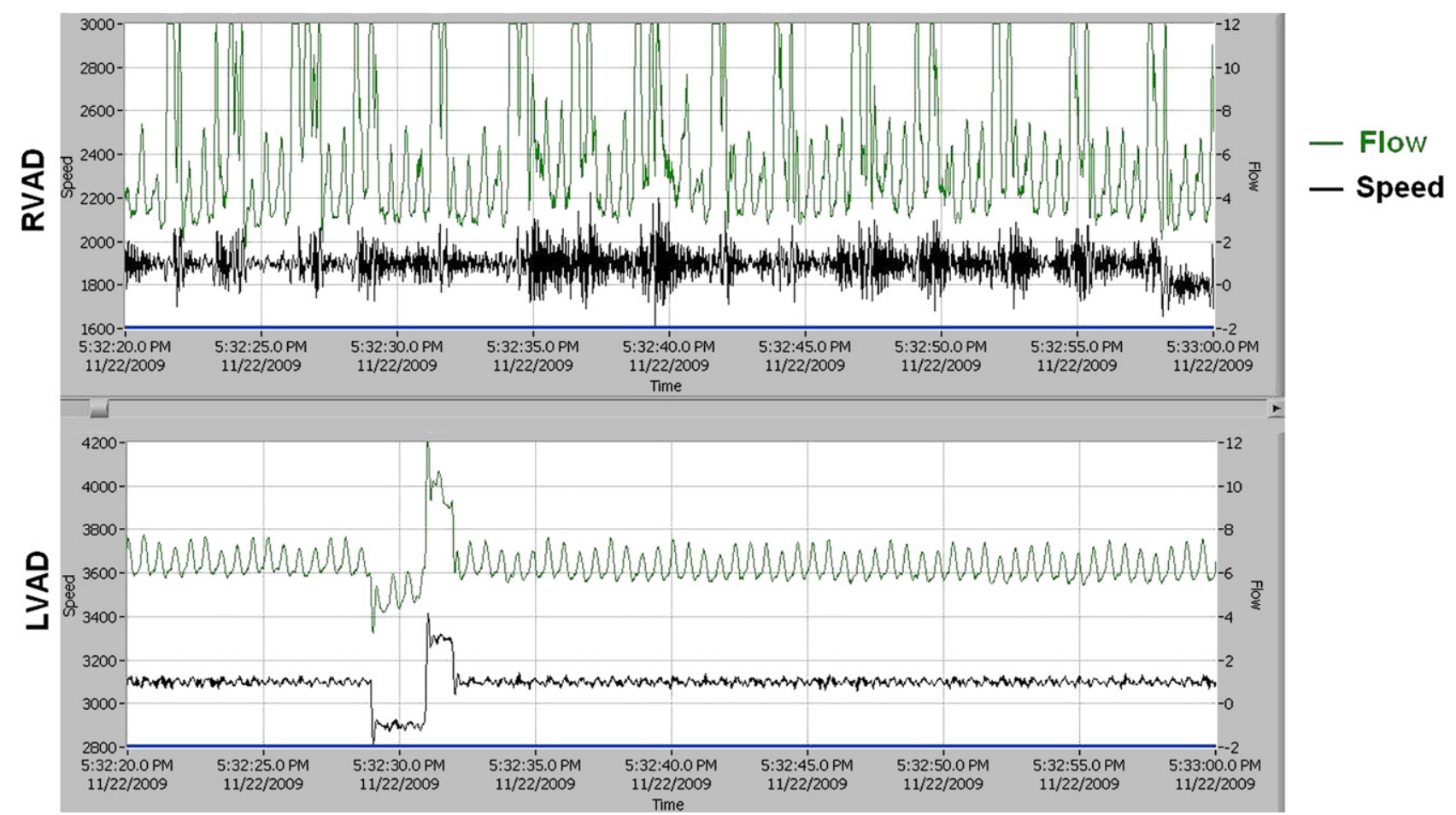

FIGURE 2. High-resolution log file of the right and left ventricular assist devices (RVAD and $L V A D)$ showing the flow and speed of both devices over a period of 40 seconds.

pump flows at inhalation (Figure 2). This resulted in changes of LA pressure (mean, 3; maximum, $12 \mathrm{~mm} \mathrm{Hg}$ ) increasing sharply at every inspiration and a constant alarm from the RVAD controller, indicating false low flow. Clinically, the patient remained well supported with the 2 devices, ambulatory and exercising to improve his physical state for future heart transplantation. On echocardiography, there was no sign of congestion or dysfunction of the RVAD. The tricuspid valve was sufficient and there was no backward flow from the device. The flow alarm of the RVAD was turned off.

Currently, the patient has been supported by both devices for 180 days without any adverse events. The patient is dependent on 2 controllers and 4 batteries. A standard protocol for anticoagulation (international normalized ratio, 2.5; clopidogrel $75 \mathrm{mg}$ ) was used. Home discharge was considered, but because of the novelty of this approach the patient is being kept in the hospital until transplantation. Concerns were raised because of the potential danger of overflowing the pulmonary circulation. Therefore, the inclusion of a resistance element (or banding) into the outflow of this device was suggested to operate the pump at higher speeds to avoid major flow changes. The device functions well without a resistance element, but the RVAD flow estimation remains erratic.

Owing to the isolated LAD occlusion, recovery of the right ventricle was expected. Probably the presence of additional significant stenosis of the dominant RCA led to persistence of right heart failure.

There have been attempts ${ }^{2}$ before to introduce rotary blood pumps into patients with biventricular failure, and we think this successful case will promote this idea even further. With minor modifications in the peripheral equipment and more research on the hemodynamics in long-term support, rotary blood pump technology may provide an alternative in destination therapy for biventricular failure.

\section{References}

1. Slaughter MS, Rogers JG, Milano CA, Russell SD, Conte JV, Feldman D, et al. HeartMate II Investigators. Advanced heart failure treated with continuous-flow left ventricular assist device. $N$ Engl J Med. 2009;361:2241-51.

2. Frazier OH, Tuzun E, Cohn WE, Conger JL, Kadipasaoglu KA. Total heart replacement using dual intracorporeal continuous-flow pumps in a chronic bovine model: a feasibility study. ASAIO J. 2006;52:145-9. 